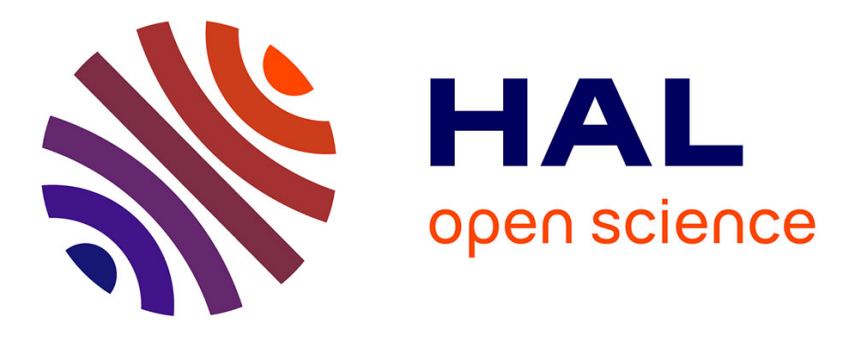

\title{
Biologically inspired incremental learning for high-dimensional spaces
}

Alexander Gepperth, Thomas Hecht, Mathieu Lefort, Ursula Körner

\section{To cite this version:}

Alexander Gepperth, Thomas Hecht, Mathieu Lefort, Ursula Körner. Biologically inspired incremental learning for high-dimensional spaces. Joint IEEE International Conference Developmental Learning and Epigenetic Robotics (ICDL-EPIROB), Sep 2015, Providence, United States. 10.1109/DEVLRN.2015.7346155 . hal-01250961

\section{HAL Id: hal-01250961 https://hal.science/hal-01250961}

Submitted on 6 Jan 2016

HAL is a multi-disciplinary open access archive for the deposit and dissemination of scientific research documents, whether they are published or not. The documents may come from teaching and research institutions in France or abroad, or from public or private research centers.
L'archive ouverte pluridisciplinaire HAL, est destinée au dépôt et à la diffusion de documents scientifiques de niveau recherche, publiés ou non, émanant des établissements d'enseignement et de recherche français ou étrangers, des laboratoires publics ou privés. 


\title{
Biologically inspired incremental learning for high-dimensional spaces
}

\author{
Alexander Gepperth, Thomas Hecht, Mathieu Lefort ENSTA ParisTech/UIIS lab \\ 828 Boulevard des Maréchaux, 91762 Palaiseau, France and \\ INRIA FLOWERS \\ 200 avenue de la Vieille Tour, 33405 Talence Cedex \\ Ursula Körner Honda Research Institute Europe $\mathrm{GmbH}$ \\ Carl-Legien-Str.30, 73076 Offenbach am Main, Germany
}

\begin{abstract}
We propose an incremental, highly parallelizable, and constant-time complexity neural learning architecture for multi-class classification (and regression) problems that remains resource-efficient even when the number of input dimensions is very high $(\geq 1000)$. This so-called projection-prediction (PROPRE) architecture is strongly inspired by biological information processing in that it uses a prototype-based, topologically organized hidden layer trained with the SOM learning rule that updates hidden layer weights whenever an error occurs. The SOM learning adapts only the weights of localized neural sub-populations that are similar to the input, which explicitly avoids the catastrophic forgetting effect of MLPs in case new input statistics are presented. The readout layer applies linear regression to hidden layer activities subjected to a transfer function, making the whole system capable of representing strongly non-linear decision boundaries. The resource-efficiency of the algorithm stems from the fact of approximating similarity in the input space by proximity in the SOM layer due to the topological SOM projection property. This avoids the storage of inter-cluster distances (quadratic in number of hidden layer) or input space covariance matrices (quadratic in input dimensions) as K-means, RBF or LWPR would have to do. Tests on the popular MNIST handwritten digit benchmark show that the algorithm compares favorably to state-of-the-art results, and parallelizability is demonstrated by analyzing the efficiency of a parallel GPU implementation of the architecture.
\end{abstract}

\section{INTRODUCTION}

Incremental learning remains a challenging issue in machine learning. While it is almost self-evident to biologists that learning should be incremental, the technical realization presents baffling difficulties. First of all, incremental learning is inherently sub-optimal when it comes to optimizing an objective (or loss) function. As one can never assume to have seen all training samples at any single point during training, optimization can only take into account the examples seen up to the present moment. Furthermore, the statistics of inputoutput relations are usually not homogeneous for any finite dataset, so incremental learning must essentially assume nonstationary input statistics at some time scale, which raises the question of how to fuse already learned aspects of a task, without destroying them, with new ones. The latter issue is a real problem for connectionist models of learning [1] and has been termed "catastrophic forgetting", and it is clear that any feasible incremental learning algorithm needs to avoid this issue. a) Biological foundations and computational modeling: As biological incremental learning has reached a high degree of perfection, we explicitly investigated the biological literature for hints as how to this might be achieved. Basing ourselves on observations from the basic sensory cortices, we noted that sensory representations seem to be prototype-based, where prototype-sensitive neurons are topologically arranged by similarity [2], [3], [4], [5]. Learning seems to act on these representations in a task-specific way, where more prototypes are allocated to sensory regions where finer discrimination is necessary [6], i.e., where more errors occur during learning. Learning is conceivably enhanced through acetylcholine release in case of task failures [7], [8], leading to higher "prototype density" in difficult regions of the sensory space. In particular, learning seems to respect and even generate topological layout of prototypes by changing only a small subset of neural selectivities [9] at each learning event, namely around those neurons that best matched the presented stimulus [5].

We model these findings by using a self-organizing map (SOM) learning to shape the feature preferences of hidden layer neurons in our architecture. SOM is a prototype-based algorithm in the sense that the hidden layer weight vectors "live" in the space of inputs in the sense that they are as close as possible to actually occurring inputs according to the SOM energy function (we use a slightly modified SOM model, see[10] that has such a globally decreasing energy function, in contrast to the original model). We model the global, taskrelated error signal by the current classification error that activates SOM learning in case of mismatch. As SOM learning attributes more prototypes to regions where many learning events occur, this will ensure that prototype density increases in difficult regions of the input space. Furthermore, SOM adaptation is stably self-terminating since no more learning will occur when no more errors are made. Inversely, when error rates increase due to the presentation of new input statistics, the hidden layer representation will become plastic until error rates subside again, when a sufficient re-adaptation has been achieved. Thus, the hidden layer represents no longer a pure data distribution but a data distribution modulated by task demands. Lastly, SOM produces a topologically organized representation of the input space, which was the reason to formulate the model in the first place, and modifies weights 


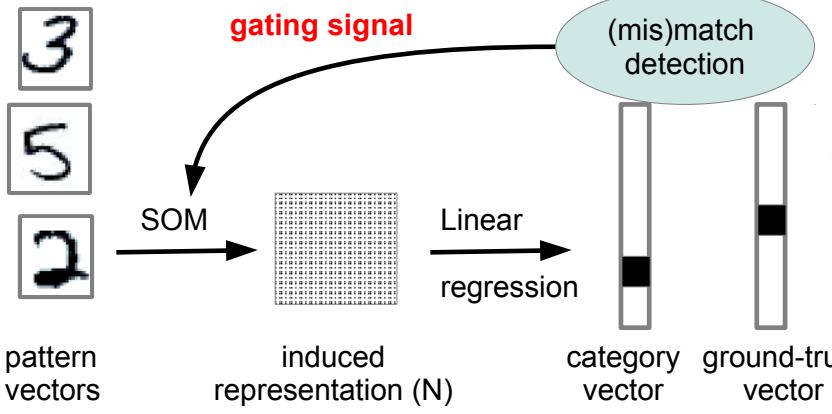

Fig. 1. Schema of the three-layer PROPRE architecture composed of input, induced and output representations. Initially there is a forward transmission step, propagating the information to the top-level of the hierarchy where it is decided whether a correct result was obtained. In case it was not, SOM weights are updated in the feedback step, thus leading to a representation of difficult samples in $N$.

only locally in case of learning (as observed in biology).

Summarizing, we have tried to incorporate as many facts about incremental learning in biology as possible while keeping the model as simple and efficient as possible. Our modeling takes place at the architectural level, leaving aside the finer details of neural modeling (rate/spike code, dynamic neuron models etc.).

b) Model properties: We propose a three-layer neural model for incremental learning that contains a topologically organized representation of prototypes in its hidden layer (termed "induced representation"), trained by the selforganized map (SOM) algorithm [11]. Due to the properties of SOMs, learning is always strictly local in the sense that only the prototypes that are similar to the best-matching one are adapted, thus avoiding catastrophic forgetting. SOM learning is activated by adverse task performance, which conversely means that learning stops once the task is acquired, thus maintaining long-term stability. Classification is performed by simple linear regression from the hidden layer towards a population-coded target vector after first applying a non-linear transfer function to all hidden layer activities.

c) Related work: Incremental learning algorithms are especially interesting for robotics applications [12], and in fact several very interesting proposals have already been made in this context [12]. An especially popular algorithm in robotics is LWPR [13], which partitions the input spaces into receptive fields (RFs), volumes that are defined by a centroid and a covariance matrix, to which separate linear models are applied. Many other incremental algorithms, reviewed in [12] partition the input space in a similar way. Although LWPR (and similar approaches that partition the input space) has been shown to be very powerful, it runs into memory problems when the product of input and output dimensionalities $K, P$ becomes large (e.g., $\geq 10000$ ). This is because each RF requires the storage of a covariance matrix of dimensionality $K^{2}$, and RFs are created independently for each output dimension, thus giving a total memory complexity of $K^{2} P$. This makes LWPR rather unsuited for problems with high input and output dimensionalities. On the other hand, RBF approaches are conceptually very close to ours in that they perform a projection onto a set of prototypes, followed by linear regression. However, incremental learning capacity is lacking on most of these models because the cluster centers ("prototypes" in our architecture) are fixed in advance, based on training data. Furthermore, RBF approaches have no notion of topology preservation when creating cluster centers; whereas our approach essentially amounts to clustering as well, the topology preserving properties of the self-organizing map model are used to approximate input space distances by distances in the projected space, which allows for efficient incremental learning.

d) Contribution of this article: In this article, we will propose a model for incremental learning that can cope with scenarios where $K M \sim 10000$ ( $K, M$ denoting input/output dimensionality) and beyond, and evaluate its performance on the well-known MNIST benchmark [14]. We explicitly evaluates the incremental aspect of learning by training on a subset of MNIST classes and subsequently adding the remaining classes. The key idea of our approach is to approximate distances in the high-dimensional input space by grid distances in the projected input space (the hidden layer of our architecture) which is a key property of the SOM algorithm we employ for this purpose. In this way, learning can be fully incremental by restricting weight changes to a small hypervolume around the current input without having to store RF centers and covariance matrices. Furthermore, the architecture we present has constant time complexity and is fully and naturally parallelizable, which we demonstrate by execution speed measurements using a separate GPU implementation ${ }^{1}$.

\section{Methods}

\section{A. The PROPRE architecture}

PROPRE is an architecture composed of different algorithmic modules, rather than an algorithm in itself. One PROPRE iteration consists of the following steps, as described in [15], where only the computation of the predictability measure $\lambda$ is changed to represent the current binary classification error:

input: new data is fed into the input representation $I$ and provided to the SOM, and a new target representation $T$ is provided projection: activity is formed in the induced representation $N$ (see Fig. 1) by projection of $I$ onto the SOM prototypes prediction: based on activity in $N$, a linear regression step is performed to produce representation $P$ which predicts class membership evaluation: a mismatch measure is computed between $P$ and $T$ update: linear regression weights are updated. SOM weights are updated only if mismatch was detected. In mathematical terms, the whole model is governed by the following equations, where we denote neural activity at position $\vec{y}=(a, b)$ in a $2 \mathrm{D}$ representation $X$ by $z^{X}(\vec{y}, t)$ and weight matrices for SOM and LR, represented by their

\footnotetext{
${ }^{1}$ All simulation code will be made available for download
} 
line vectors attached to target position $y=(a, b)$ by $w_{\vec{y}}^{\text {SOM}}$ :

$$
\begin{aligned}
\tilde{z}^{N}(\vec{y}, t) & =\left|w_{\vec{y}}^{\mathrm{SOM}}(t)-z^{I}(t)\right| \\
\bar{z}^{N}(\vec{y}, t) & =g_{\kappa}\left(\tilde{z}^{N}\right) \\
z^{N}(\vec{y}, t) & =\operatorname{TF}\left(\bar{z}^{N}(\vec{y}, t)\right) \\
z^{P}(\vec{y}, t) & =w_{\vec{y}}^{\mathrm{LR}}(t) \cdot \mathrm{TF}\left(z^{N}(t)\right) \\
\lambda(t) & =0 \text { if } \operatorname{argmax}_{\vec{y}} z^{P}(\vec{y}, t)=\operatorname{argmax}_{\vec{y}} z^{T}(\vec{y}, t) \\
1 & \text { else } \\
w_{\vec{y}}^{\mathrm{LR}}(t+1) & =w_{\vec{y}}^{\mathrm{LR}}(t)+2 \epsilon^{\mathrm{LR}} z^{I}(t)\left(z^{P}(t)-z^{T}(t)\right) \\
w_{\vec{y}}^{\mathrm{SOM}}(t+1) & =w_{\vec{y}}^{\mathrm{SOM}}(t)+\lambda(t) \epsilon^{\mathrm{SOM}} g_{\sigma}\left(\vec{y}-\vec{y}^{*}\right)\left(z^{I}-w_{\vec{y}}^{\mathrm{SOM}}\right) \\
\kappa(t+1) & =0.999 \kappa(t)+0.001 \max _{\vec{y}} \tilde{z}^{N}(\vec{y}, t)
\end{aligned}
$$

where $g_{s}(x)$ is a zero-mean Gaussian function with standard deviation $s$ and $\vec{y}^{*}$ denotes the position of the best-matching unit (the one with the highest activity) in $N$. In accordance with standard SOM training practices, the SOM learning rate and radius, $\epsilon^{\mathrm{SOM}}$ and $\sigma$, start at $\epsilon_{0}, \sigma_{0}$ and are exponentially decreased in order to attain their long-term values $\epsilon_{\infty}, \sigma_{\infty}$ at $t=T_{\text {conv }}$. In order to convert euclidean distance between a prototype vector and the input into a similarity score in the interval $[0,1]$, we pass the "naked" distances $\tilde{z}^{N}$ through a zero-mean Gaussian function with a standard deviation $\kappa(t)$ that adapts to the average maximal distance found in the whole induced representation. This amounts to determining, over time, the expected maximal distance to which a small score should be assigned, whereas the highest score would always be assigned to a distance of 0 . TF represents a monotonous non-linear transfer function, $\mathrm{TF}:[0,1] \rightarrow[0,1]$ which we model as follows with the goal of maintaining the BMU value unchanged while gradually and nonlinearly suppressing all other values:

$$
\begin{aligned}
m_{0} & =\max _{\vec{y}} \bar{z}^{N}(\vec{y}, t) \\
m_{1} & =\max _{\vec{y}}\left(\bar{z}^{N}(\vec{y}, t)\right)^{20} \\
\operatorname{TF}\left(\left(\bar{z}^{N}(\vec{y})\right)\right. & =m_{0} \frac{\left(z^{N}(\vec{y})\right)^{20}}{m_{1}}
\end{aligned}
$$

A softmax function would have done the trick as well, but we avoid this in order not to calculate too many exponentials.

\section{B. The MNIST handwritten digit database}

For all experiments, we use he publicly available MNIST classification benchmark as described in [14]. It contains 10 classes, corresponding to the 10 handwritten digits from "0" to "9", see also Fig. 1, and comes separated into a well-defined train set and a smaller test set. Each sample has a dimensionality of $K=28 \times 28=784$. From the MNIST benchmark, we extract several subsets of classes: $D_{\overline{0}}$ containing the digits from 1 to 9 , and $D_{0}$ containing just the digit "0". Analogously, we create $D_{\overline{1}}, D_{\overline{2}}, D_{1}$ and $D_{2}$. Each set $D_{x}$ is again split into training and test sets $D_{x}^{\text {train }}, D_{x}^{\text {test }}$ to measure generalization performance, the split being made according to whether a certain sample belongs to the MNIST train or test set. For training and evaluating performance on all digits, we also create the sets $D_{0-9}^{\text {train }}, D_{0-9}^{\text {test }}$ which correspond to the original MNIST train and test sets.

\begin{tabular}{|cc|ccccc|}
\hline$n$ & $\epsilon_{\infty}$ & 2 & 1 & 0.5 & 0.1 & 0.05 \\
\hline 10 & 32.9 & 17.9 & 10.3 & 8.5 & 8.6 \\
20 & 17.0 & 10.0 & 6.3 & 5.5 & 5.4 \\
30 & 12.8 & 7.2 & 5.5 & 4.8 & 4.8 \\
50 & 7.9 & 5.4 & 4.8 & 4.4 & 4.6 \\
\hline
\end{tabular}

TABLE I

BASELINE NON-INCREMENTAL PERFORMANCE EVALUATION OF PROPRE ON MNIST DATA WHILE VARYING THE MINIMAL SOM NEIGHBOURHOOD RADIUS AND THE HIDDEN LAYER SIZE. GIVEN ARE THE ERRORS ON THE MNIST TEST SET IN PERCENT.

\section{EXPERIMENTS}

We use the following fixed parameters for PROPRE: $\epsilon^{\mathrm{LR}}=$ $0.001, \epsilon_{0}=0.5, \sigma_{0}=0.3 n, T_{1}=50000, T_{\text {conv }}=100000$, $\epsilon_{\infty}=0.001$ and $\sigma_{\infty}=0.5$. Both SOM and LR weight matrices are initialized to random uniform values between -0.001 and 0.001 . No preprocessing is performed on the 28x28-dimensional MNIST input vectors. Training examples are always randomly and uniformly drawn from the current training set.

\section{A. Baseline performance measurement}

In order to establish a baseline performance that demonstrates the principal capability of the PROPRE architecture to solve the classification problem posed by MNIST, we first train and evaluate the PROPRE architecture for $10^{6}$ iterations on $D_{0-9}$. Modulation of SOM learning is turned off by setting $\lambda(t) \equiv 1$ in eqns.(1) as this is not in incremental learning task. The performance thus obtained is to be compared to offline, batch-type algorithms. In the case of PROPRE, this would be other three-layer architectures such as multilayer perceptron or RBF networks. In particular, a goal of this experiment is to find a value of $\epsilon_{\infty}$ that will give maximal performance in this non-incremental setting. This is an important point as the capacity of the hidden layer to represent inputs as closely as possible is intimately tied to this parameter: the smaller it is, the smaller will be the average prototype-input distance expressed by the similarity score of the BMU, and it can be reasonably speculated that this is in turn related to classification performance of the linear regression readout. We will at the same time vary the hidden layer size $n^{2}$, again with the goal of maximizing classification performance, where it is again reasonable to suppose that bigger hidden layers will give better classification as the inputs can be approximated with a higher overall resolution.

The results of this set of experiments are summarized in Table I. The show mainly two things:

- Smaller $\epsilon_{\infty}$ leads to better classification performance

- Bigger hidden layer sizes lead to better classification performance

Especially the latter result, while not really surprising, is interesting, as it suggests that in the case of the PROPRE architecture, we do not suffer from the problem of choosing a correct hidden layer size as in the case of multilayer perceptrons (MLPs). As the hidden layer projections are shaped by an energy-based variant of the generative SOM learning 


\begin{tabular}{|c|ccc|}
\hline train dataset $D_{x}^{\text {train }}$ & $D_{0}^{\text {train }}$ & $D_{1}^{\text {train }}$ & $D_{2}^{\text {train }}$ \\
\hline test dataset & 5.95 & 6.11 & 5.75 \\
$D_{\bar{x}}^{\text {test }}$ at $t=300.000$ & 3.57 & 0.96 & 6.5 \\
$D_{x}^{\text {test }}$ at $t=350.000$ & 6.41 & 6.75 & 6.19 \\
$D_{\bar{x}}^{\text {test }}$ at $t=350.000$ & $5.82(4.8)$ & $5.96(4.8)$ & $6.21(4.8)$ \\
$D_{0-9}^{\text {test }}$ at $t=350.000$ & & \\
\hline
\end{tabular}

TABLE II

INCREMENTAL LEARNING PERFORMANCES WHEN TRAINING ON 9 MNIST CLASSES AND ADDING THE REMAINING ONE AFTERWARDS, FOR THREE DIFFERENT CHOICES OF THE LATTER, NAMELY "0", "1" AND "2". FOR

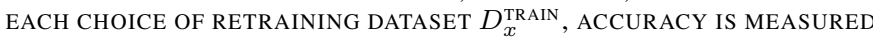
FOR THREE DIFFERENT TEST SETS: $D_{x}^{\text {TEST }}, D_{\bar{x}}^{\text {TEST }}$ AND $D_{0-9}^{\text {TEST }}$. FOR THE

LATTER, THE FIGURE IN PARENTHESES IS THE BASELINE PERFORMANCE, THAT IS, FOR THE CASE OF NON-INCREMENTAL LEARNING.

algorithm, it is intuitively clear that having more prototypes implies a more precise representation of inputs which in turn favors classification performance. As a last point, we found, again not very surprisingly, that the application of a nonlinear transfer function to the SOM similarity scores computed according to Sec. II-A is essential for acceptable performance. With purely linear transfer functions, performance drops of more than $10 \%$ occur where precise figures depend on hidden layer size $n^{2}$.

\section{B. Incremental learning performance}

We conduct several experiments designed to measure the capability to perform incremental learning. To this effect, we let the architecture converge on the datasets $D_{\overline{0}}^{\text {train }}, D_{\overline{1}}^{\text {train }}$ or $D_{\overline{2}}^{\text {train }}$ for 300.000 iterations, keeping all timing parameters like $T_{1}$ and $T_{\text {conv }}$ unchanged from the baseline experiment, and performing the same reduction of learning rates and neighbourhood radius. The modulation factor is kept at $\lambda(t) \equiv 1$ for $t<300.000$ in order to have a defined starting point, and is determined according to eqns.(1) for $t \geq 300.000$. From $t=300.000$ onwards, we present one of the complementary datasets $D_{0}^{\text {train }}, D_{1}^{\text {train }}$ or $D_{2}^{\text {train }}$ for 20000 iterations, followed by a phase of 30.000 iterations where SOM plasticity is turned off $(\lambda(t) \equiv 0)$ in order to let linear regression weights "catch up" with the changes to the hidden layer selectivities. At all times, we can measure model performance on any of the corresponding test sets.

e) Results: The numerical results of these experiments, conducted for $\sigma_{\text {inf }}=0.05$, are given in Tab. II. They clearly show that incremental learning is successful, as the newly added class is well learned while performance on the "old" classes is retained with little change, which is also reflected in the fact that the overall error on $D_{0-9}^{\text {test }}$ increases only insignificantly. As all classes are present in equal frequency in the MNIST benchmark, an inferior performance on the newly added class could raise error rates by up to 10 percent which is not observed.

We observe what happens when the modulation factor $\lambda(t)$ in eqns.(1) is kept fixed at lambda $\equiv 1$ meaning that the SOM weights are updated at every iteration, regardless of errors in classification. Although errors on the new class drop very quickly, errors on the "old" classes rise much more quickly and unpleasantly than in the case where $\lambda(t)$ is determined from current classification accuracy according

\begin{tabular}{|c|cccc|}
\hline time & $10 \times 10$ & $20 \times 20$ & $30 \times 30$ & $50 \times 50$ \\
\hline GPU & 15 & 19 & 27 & 53 \\
CPU & 115 & 457 & 1037 & 2903 \\
\hline
\end{tabular}

TABLE III

EXECUTION TIME MEASUREMENTS IN SECONDS PER $10^{4}$ ITERATIONS OF PROPRE FOR GPU AND CPU IMPLEMENTATIONS. IT CAN BE OBSERVE THAT GPU EXECUTION TIMES SCALE (MUCH) LESS THAN LINEARLY IN THE CONSIDERED RANGE OF HIDDEN LAYER SIZES, WHEREAS THE CPU IMPLEMENTATION SCALES ALMOST EXACTLY IN A LINEAR FASHION.

to eqns.(1). This is because eqns.(1) update SOM weights only when misclassifications occur, or conversely, do not adapt anything when classifications are accurate. When presenting a new class, initially all classifications will be incorrect and strong adaptation occurs. After having learned a sufficiently good representation in the hidden layer, adaptation of SOM weights largely stops, which protects the old classes regardless of how long the new class is actually presented. Briefly put, the learning architecture we presents adapts its hidden layer selectivities only as much as necessary and no more.

We furthermore observe that the value of $\sigma_{\infty}$ seems to control the incremental learning capacity of the architecture: if it is too large, adaptation is too fast and the old classes will be completely overwritten before all samples of the new class have even been fully presented. conversely, if it is too small, changing input statistics are incorporated too slowly to play a role within the considered 20.000 retraining iterations. This is natural since the SOM algorithm guarantees a graceful decay whose time scale is however controlled by $\sigma_{\infty}$. Fig. 2 shows the effect of large, small and just correct values for $\sigma_{\infty}$.

\section{Parallelization and complexity issues}

Apart from the very favorable time and memory complexity of the PROPRE architecture, all of its component parts can be very efficiently parallelized. Here we focus predominantly on the SOM layer as it produces the highest computational burden. We can deduce from eqn. (1) that both the projection as well as the weight adaptation part can be performed in parallel for each output neuron. This is evident for the projection step that produces $z^{N}$, as well as for the weight adaptation step that just makes use of the activities $z^{N}$ computed during the projection step but not of the weight vectors (SOM prototypes) of other hidden layer neurons. In fact, in a parallel implementation we can go even further and stop weight adaptation for a particular hidden layer neuron when it is too far away from the best-matching unit (BMU), i.e., when the neighbourhood function $g_{\sigma}$ falls below a certain threshold for which we have taken a very conservative value of $10^{-2}$. Since the weight change becomes negligible by multiplying with the current learning rate $(<<1)$ this is a very justified approximation. For a purely CPU-based implementation without parallelism, this enormously speeds up computations when the neighbourhood radius has converged to its long-term level of $\sigma_{\infty}$, as effectively only a very small fraction of the total set of prototypes is adapted at each time step, namely those that are very close to the BMU. For a parallel GPU implementation, the benefit of this approximation depends 

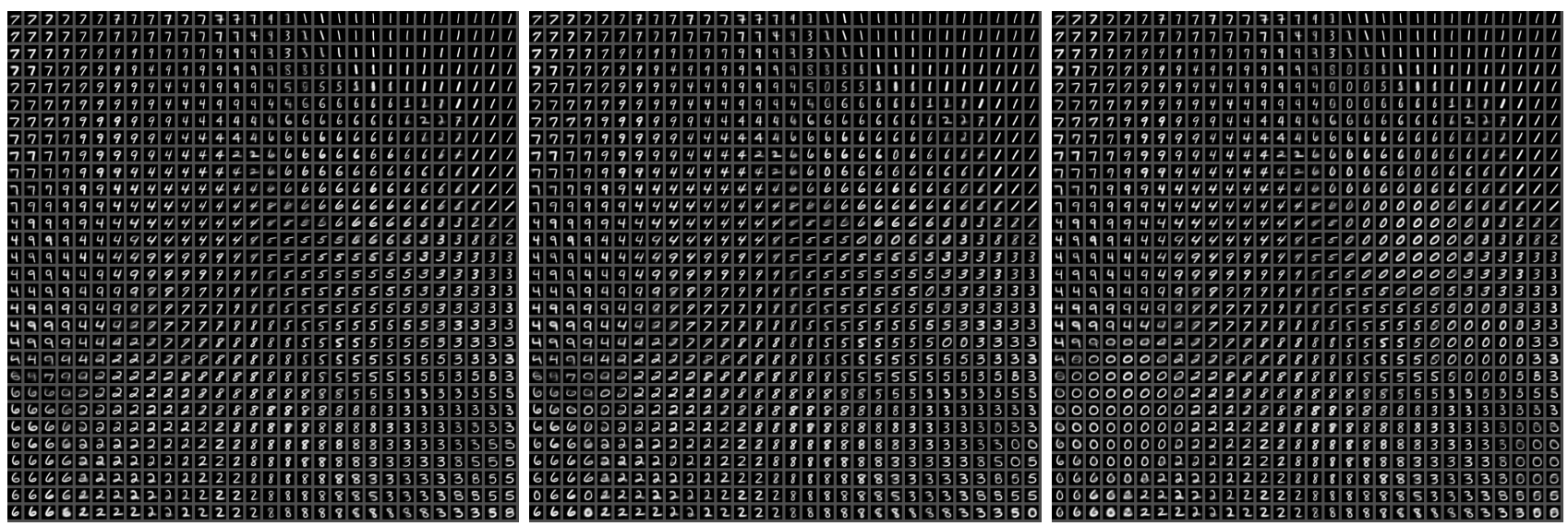

Fig. 2. Effect of incremental learning on SOM prototypes. Shown is a 30x30 grid of prototypes in the form of little images of size $28 \times 28$, the size of one MNIST sample. Left: SOM converged on $D_{\overline{0}}^{\text {train }}$ at $t=300.000, \sigma_{\infty}=0.05$. The similarity-based ordering of prototypes in the typical fashion of a SOM is well discernible. Middle: SOM with addition of the new class " 0 " at $t=320.000$. We can clearly observe that the new class is inserted into the SOM at positions where it most closely matches existing prototypes (e.g., bottom left corner), and that prototypes dissimilar to the new "0" class (e.g., class "1" in the upper right corner) are not affected at all. Right: SOM with addition of new class at $t=320.000$, with a different value of $\sigma_{\infty}=0.75$. We observe that insertions are now much more spatially organized by similarity, and that the prototypes representing the new " 0 " class are much more frequent, showing that more of the "old" knowledge has been overwritten.

strongly on the actual way of distributing computations on the available multiprocessors, but is less pronounced for modern GPUs with high parallelism, because then each parallel process computes very little (typically 1-2 additions) and the difference of aborting or executing it is small.

The GPU implementation was done using CUDA on a GeForce GTX 570 graphics card (a rather old model) on a rather old computer (Pentium Quad-Core of $2 \mathrm{GHz}$ running Linux). The actual implementation was performed in Python using the pyCUDA library[16]. The reference implementation was done in Python as well using the numpy library[17] which is optimized for vectorization, so the approximation mentioned above was not employed. Obtained execution times are summarized in Tab. III. They show a very large performance difference between CPU and GPU implementations, on the one hand in absolute terms and on the other hand in terms of scaling: whereas CPU execution times scale exactly with the change in hidden layer size, the GPU execution times grow much more slowly and allow therefore to simulate much larger architectures. It is clear that this behavior will saturate at some point, but as the whole point of this experiments was to show to what extent and with what facility PROPRE can be parallelized, this is not a crucial issue.

\section{DISCUSSION}

f) Complexity: Incremental learning using the PROPRE architecture comes at constant time complexity; this is in contrast to conventional incremental learning algorithms such as LWPR which allocate "receptive fields" at runtime as needed, and whose time complexity is linear in the number of these structures. In a sense, PROPRE "pre-allocates" a certain number of "receptive fields" and uses them as well as it can, where having more receptive fields means better classification accuracy. Denoting input dimensionality, hidden layer size and output layer size (number of classes) by $\mathrm{K}, \mathrm{N}$ and $\mathrm{P}$, the memory complexity of PROPRE is roughly
$(K N+N P)=N(K+P) \approx N K$. For a pure RBF classifier with $\mathrm{N}$ cluster centers, the memory complexity would be $N(K+P) \approx N K$ as well. However, if cluster centers should need to be updated in an efficient fashion, it will be necessary to store a matrix of inter-cluster distances so that each new sample can update the clusters to which it is nearest in the input space. This matrix will have $N^{2}$ entries, making the total memory complexity $N(N+K+P \approx N(N+K)$ which can be formidable for a large number of clusters. For the LWPR algorithm, the storage of receptive fields that are defined in the input space requires approximately $P N\left(K+4 K^{2}\right) \approx 4 P N K^{2}$ which becomes prohibitive for large input dimensionalities $K$. The factor 4 in the last expression comes from the storage of sufficient data statistics along with each receptive field as detailed by [18].

g) Is this really incremental learning?: in this article, we show how we can, in an additional training step, teach new things to our architecture without forgetting too much of previously learned knowledge. Where forgetting happens, it has a certain graceful decay property that is characteristic for the SOM model. However, in order to teach "new tricks" to the architecture, we perform a dedicated incremental learning procedure that is different from the initial learning procedure: fist, we present the new concept in the form of examples, and perform a subsequent linear regression retraining where SOM learning is deactivated. Each time something new (e.g., a class) is added, this step has to be repeated. This does not pose problems in practice, but from a conceptual point of view it would be much more elegant to perform incremental learning identically to the initial learning step. Already, the term "incremental learning" is not well defined in the literature, but if we stick to the terms defined in [12], we can say that our method is incremental but not fully online.

h) Incremental vs. non-incremental performance: We observe in all experiments that incremental learning incurs a slight cost in the form of a departure from the non- 
incremental version of the PROPRE architecture. This is not very surprising, first of all as the online linear regression we use to read out hidden layer activities may not yet be fully converged, but mainly because the addition of a new class to an already learned model is inherently sub-optimal (initial learning does not know at all about the new classes).

i) Influence of $\sigma_{\infty}$ : We found a strong impact of $\sigma_{\infty}$ on map formation in the hidden layer which in turn strongly influences classification accuracy. As suggested by Fig. 2, this parameter also governs the way incremental learning adapts SOM prototypes. We believe that further work will be necessary to elucidate the precise role of this parameter.

\section{CONCLUSION}

We have presented an algorithm for resource-efficient incremental learning that draws its efficiency from principles of biological information processing and showed that it can easily handle data dimensionalities of 750 entries. We showed that it compares favorably with the state-of-the-art on a standard machine learning benchmark (MNIST) in its non-incremental form, and that good classification accuracy persists when training it incrementally by adding classes to a trained model using the same benchmark. The algorithm is self-limiting and destroys old knowledge only "on demand", due to misclassifications. Due to neural design principles, the whole architecture can be very easily parallelized, obtaining performance gains up to a factor of 8 , along with a much nicer scaling behavior when the hidden layer size is increased. Future work will include "deep" PROPRE architectures, namely investigating how the incremental learning capacity can be maintained in such an architecture, as well as efforts to make the PROPRE architecture fully online, which means that training and retraining steps should be conducted in the exact same fashion, thus assuring maximal simplicity and applicability in many different applied scenarios where autonomous, uncontrolled learning is required.

\section{REFERENCES}

[1] Ian J Goodfellow, Mehdi Mirza, Xia Da, Aaron Courville, and Yoshua Bengio. An empirical investigation of catastrophic forgeting in gradientbased neural networks. arXiv preprint arXiv:1312.6211, 2013.

[2] Keiji Tanaka. Inferotemporal cortex and object vision. Annual review of neuroscience, 19(1):109-139, 1996.

[3] David A Leopold, Igor V Bondar, and Martin A Giese. Norm-based face encoding by single neurons in the monkey inferotemporal cortex. Nature, 442(7102):572-575, 2006.

[4] David A Ross, Mickael Deroche, and Thomas J Palmeri. Not just the norm: Exemplar-based models also predict face aftereffects. Psychonomic bulletin \& review, 21(1):47-70, 2014.

[5] Cynthia A Erickson, Bharathi Jagadeesh, and Robert Desimone. Clustering of perirhinal neurons with similar properties following visual experience in adult monkeys. Nature neuroscience, 3(11):1143-1148, 2000.

[6] Daniel B Polley, Elizabeth E Steinberg, and Michael M Merzenich Perceptual learning directs auditory cortical map reorganization through top-down influences. The journal of neuroscience, 26(18):4970-4982, 2006.

[7] Norman M Weinberger. The nucleus basalis and memory codes: Auditory cortical plasticity and the induction of specific, associative behavioral memory. Neurobiology of Learning and Memory, 80(3):268 - 284, 2003. Acetylcholine: Cognitive and Brain Functions.

[8] Michael E Hasselmo. The role of acetylcholine in learning and memory. Current opinion in neurobiology, 16(6):710-715, 2006.
[9] Edmund T Rolls, GC Baylis, ME Hasselmo, and V Nalwa. The effect of learning on the face selective responses of neurons in the cortex in the superior temporal sulcus of the monkey. Experimental Brain Research, 76(1):153-164, 1989.

[10] Tom Heskes. Energy functions for self-organizing maps. Kohonen maps, pages 303-316, 1999.

[11] T Kohonen. Self-organized formation of topologically correct feature maps. Biol. Cybernet., 43:59-69, 1982.

[12] Olivier Sigaud, Camille Salaün, and Vincent Padois. On-line regression algorithms for learning mechanical models of robots: a survey. Robotics and Autonomous Systems, 59(12):1115-1129, 2011.

[13] Sethu Vijayakumar, Aaron D'souza, and Stefan Schaal. Incremental online learning in high dimensions. Neural computation, 17(12):26022634, 2005.

[14] Y. LeCun, L. Bottou, Y. Bengio, and P. Haffner. Gradient-based learning applied to document recognition. In Intelligent Signal Processing, pages 306-351. IEEE Press, 2001.

[15] A Gepperth. Efficient online bootstrapping of representations. Neural Networks, 2012.

[16] Andreas Klöckner, Nicolas Pinto, Yunsup Lee, B. Catanzaro, Paul Ivanov, and Ahmed Fasih. PyCUDA and PyOpenCL: A Scripting-Based Approach to GPU Run-Time Code Generation. Parallel Computing, 38(3):157-174, 2012

[17] Stefan van der Walt, S. Chris Colbert, and Gael Varoquaux. The numpy array: A structure for efficient numerical computation. Computing in Science \& Engineering, 13(2), 2011.

[18] Sethu Vijayakumar Stefan Klanke and Stefan Schaal. A library for locally weighted projection regression. Journal of Machine Learning Research (JMLR), 9:623-626, 2008. 